\title{
Analysis of the performance of an evolutionary computation algorithm in the identification of geomechanical parameters in underground works
}

\author{
T. Miranda \& A. Gomes Correia \\ C-TAC, Department of Civil Engineering, University of Minho, Guimarães, Portugal \\ J. Sena-Cruz \\ ISISE, Department of Civil Engineering, University of Minho, Guimarães, Portugal
}

L. Costa

Algoritmi, Department of Production and Systems, University of Minho, Guimarães, Portugal

D. Dias

Grenoble Alpes University, LTHE, Grenoble, France

\begin{abstract}
This paper addresses the performance analysis of an optimization algorithm from the field of evolutionary computation, namely an Evolution Strategy, in back analysis to evaluate the geomechanical parameters of the formation surrounding an underground structure. The algorithm is first tested using a synthetic case of a tunnel excavation. In this case, different scenarios are considered through a parametric study. Then the algorithm is tested using real data from the excavation of an underground structure built in the North of Portugal using a 3D model. The results show that the Evolution Strategy algorithm is robust in the identification of geomechanical parameters related to underground engineering.
\end{abstract}

\section{INTRODUCTION}

In back analysis, field measurements are used together with models to calibrate input parameters (geomechanical, stress state, etc...) matching, under a defined tolerance, predicted with observed measures. Normally, an iterative procedure is needed to find the best set of parameters through the minimization of an error function that measures the difference between real and computed quantities. For the minimization task optimization algorithms are used and this is a main issue to obtain the best set of parameters.

In geotechnics, two main types of algorithms have been used in back analysis: algorithms from the field of classical optimization theory such as the Simplex, the Newton-Raphson or gradient methods; and Evolutionary optimization algorithms like Genetic Algorithms (GA), Evolution Strategies (ES), Simulated Annealing, etc. (Moreira et al., 2013).

Classical algorithms present a satisfactory performance in smooth-shaped error functions, with a clearly defined and unique minimum (Miranda, 2007). However, the error function topology is normally complex and the uniqueness of the solution cannot be guaranteed since many local minima may occur. Hence, this kind of algorithms are most of the times limited to simpler models and a reduced number of parameters to identify (Moreira et al., 2013).

Recently, global optimization algorithms from the field of Evolutionary Computation have been used to overcome the limitations of classical algorithms with special emphasis to the GA (Levasseur et al., 2010; Miranda et al., 2011; Papon et al., 2012). Both GA and ES are identical with respect to their major working scheme. Both rely upon the collective learning paradigm gleaned from natural evolution and implement the principles of "population", "mutation", "recombination" and "selection". However, they exhibit significant differences with respect to the details of the selection scheme, the encoding of the variables to optimize and, especially, the selfadaptation of the internal parameters during the optimization process (Hoffmeister and Bäck, 1991).

The main limitation of these algorithms is that they need a substantial amount of iterations to find acceptable results, which can be, in some cases, prohibitive if large numerical models are used. However, the advances in the coding of the algorithms, more powerful computers and parallel and distributed computing can help mitigating this drawback.

This work focuses on the performance analysis of an ES in back analysis of geomechanical parameters since this type of algorithm has been scarcely used in geotechnics. For this purpose the ES is tested using a synthetic case of a tunnel excavation and data from a real case study. For the synthetic case, geomechanical parameters were attributed to the rock mass in order to obtain the "real" displacements. Then, these displacements were used together with the algorithm to back analyse the parameters of interest.

For the real case a back analysis study of geomechanical parameters was carried out in order to reduce uncertainties, using a 3D model of a power station built in the North of Portugal. Monitoring data was scarce since only one extensometer was available. Back analysis in this case will raise considerable problems of nonuniqueness so the algorithm was tested in difficult conditions. 


\section{EVOLUTION STRATEGIES}

ES algorithms were firstly developed by Rechenberg (1973). In these algorithms, the search starts from a population of individuals, in general, created at random. A new generation of individuals is generated by genetic operators constituting the offspring population. The fitness of the individuals is based on the results of the error function and constraints. It should be noted that no derivatives or other auxiliary knowledge is needed.

ES work directly with the real representation of the decision variables (in this case a set of geomechanical parameters) in which an individual is a vector of real numbers (the decisions variables) and represents a potential solution for the optimization problem.

After the first simpler versions of the ES, Rechenberg developed the $(\mu+\lambda)$-ES, that in a given generation, a population of $\mu$ parents generates $\lambda$ offspring by mutation. Then the $\mu$ parents plus $\lambda$ offspring are sorted according to their fitness value and finally the $\mu$ parents for next generation are the best individuals of the $\mu$ parents plus the $\lambda$ offspring (i.e., the selection takes place between the $\mu+\lambda$ members).

Mutation is a genetic operator that creates new points by adding random normal distributed quantities to the parent (vector of decision variables) in a process called Gaussian mutation. It is important to note that, for each decision variable, an individual standard deviation can be used controlling the step sizes.

During the search, the standard deviations (or step sizes for mutation) are adapted and this is one of the most promising features of the ES. They are updated during the process using different rules and selfadaptation schemes which enhance its performance (Rechenberg, 1994).

Later, Schwefel (1995) has reported a remarkable acceleration in the search process, as well as, the facilitation of self-adaptation of parameters by introducing a recombination operator. Basically, it consists on, before mutation, to recombine a set of chosen parents to find a new solution. Thus, the nomenclature for the ES was extended, and ES with recombination are usually referred as $(\mu / \rho+\lambda)$-ES. In the present study this type of strategy was used. The stopping criterion was set as a maximum number of 300 calculations.

\section{PARAMETRIC STUDY OF BACK ANALYSIS}

\subsection{Back analysis procedure and numerical model}

The parametric study of back analysis was carried out through the application of the ES to the identification of geomechanical parameters in a synthetic case of a tunnel excavation using a 3D numerical model. The algorithm was programmed in Matlab 7.9 (2009) and connected to the numerical model to perform the iterative process. The used error function was a least square one.

The numerical model was developed with FLAC3D (Itasca, 2005) and it is a $20 \mathrm{~m}$ length tunnel composed by a $4 \mathrm{~m}$ radius arch and a vertical wall with the same span. The support system consists of $0.2 \mathrm{~m}$ thick shotcrete simulated by shell elements with linear elastic isotropic behaviour, with a Young modulus of $20 \mathrm{GPa}$ and a Poisson ratio $(v)$ of 0.2 .

The construction process begins with the excavation of the tunnel arch in a $3 \mathrm{~m}$ length followed by the application of the shotcrete on the arch, then the remaining part of the tunnel is excavated and finally the shotcrete is applied in the walls of the tunnel.

In a first stage, the formation surrounding the tunnel was simulated using an elastic constitutive model. The values of the initial geomechanical parameters used to produce the "real values" of the monitoring data were: deformability modulus (E) of $2 \mathrm{GPa}$ and a Poisson coefficient of 0.1. A gravitational stress state was considered with a horizontal to vertical stress ratio $\left(\mathrm{K}_{0}\right)$ of 0.8 in both horizontal directions. In a second stage, calculations were performed to identify the parameters of the MohrCoulomb failure criterion with non-associated flow rule. The adopted values for cohesion (c') and friction angle $\left(\phi^{\prime}\right)$ were, respectively, $50 \mathrm{kPa}$ and $32^{\circ}$. The low values of the strength parameters were set in order to induce considerable yielding zones in the model to check the influence of increased nonlinearity of the back analysis process in the behavior of the algorithm.

\subsection{Results}

The monitoring plan set in this theoretical example was composed by a total of four displacement measurements, namely: vertical displacements at the surface and in the arch and floor of the tunnel; and horizontal displacement in the wall of the tunnel. In the parametric study, several cases were analysed in order to evaluate the capabilities of the algorithm in different conditions (Table 1).

For the elastic model the influence of the number and location of measurements was evaluated. For case 1 four displacement measurements were considered. The ES converged to the correct values regardless the interval range of the parameters. The topology of the error function presents an almost convex configuration, in which the global minimum correspondent to the optimal solution is clearly defined (Figure 1). This configuration turns convergence faster and easier for the algorithms. 
Table 1. Analysed cases in the parametric study

\begin{tabular}{lllll}
\hline Model & Case & $\begin{array}{l}\text { Geom. } \\
\text { Par. }\end{array}$ & Monitored displacements \\
\hline & & & No & Location \\
\hline Elastic & 1 & E, $\mathrm{K}_{0}$ & 4 & arch, wall, surf., floor \\
& 2 & E, $\mathrm{K}_{0}$ & 2 & arch, wall \\
& 3 & E, $\mathrm{K}_{0}$ & 2 & wall, surface \\
& 4 & E, $\mathrm{K}_{0}$, & 2 & arch, surface \\
Plastic & 5 & E, c', $\phi^{\prime}$ & 4 & arch, wall, surf., floor \\
& 6 & E, c', & 4 & arch, wall, surf., floor \\
& 7 & E, $\phi^{\prime}$, & 4 & arch, wall, surf., floor \\
& 8 & c', $\phi$ & 4 & arch, wall, surf., floor \\
& 9 & E, c',, & 2 & arch, wall \\
& 10 & c',,$\phi^{\prime}$ & 2 & arch, wall \\
& 11 & E, c', $\phi$ & 2 & wall, surface \\
\hline
\end{tabular}
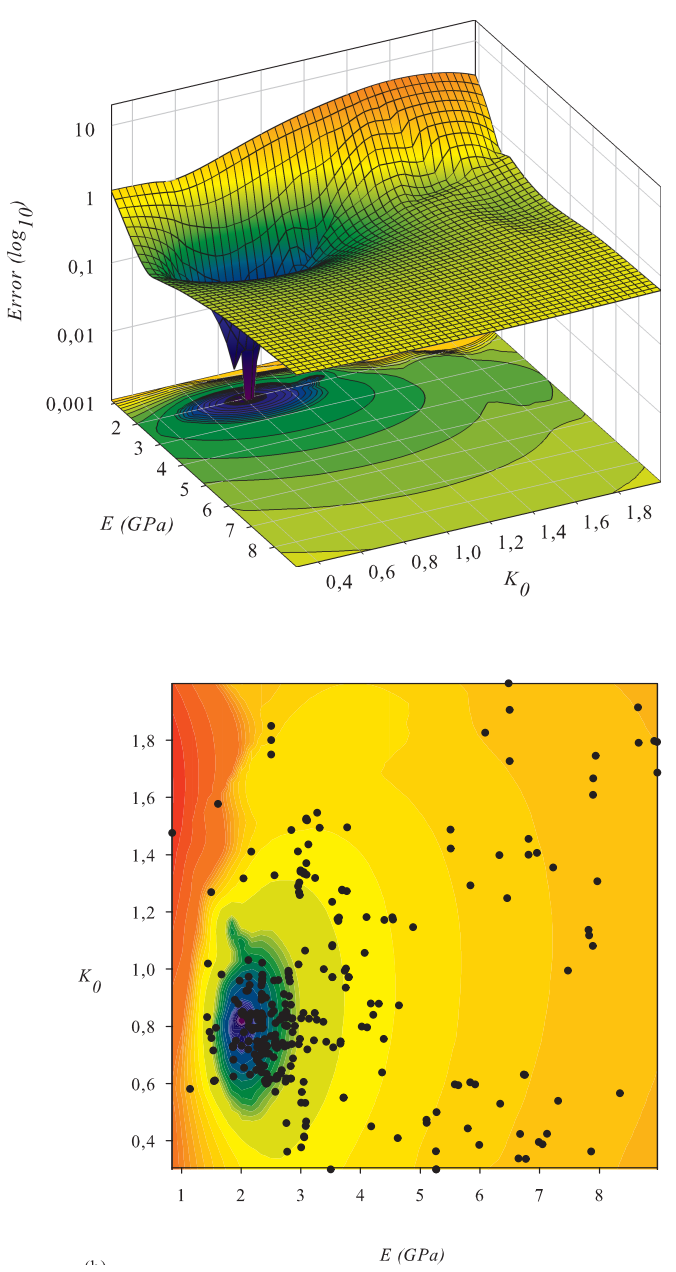

Figure 1. Topology of the error function for case 1.

In cases 2 to 4 only two measurements were considered. Cases 2 and 3 use vertical and horizontal displacements while case 4 represents the case of using two measurements with the same direction.

The algorithm converged to the correct solution in cases 2 and 3. The topology of the error functions for cases 2 and 3 are similar to the one obtained in case 1 . In case 4 the solution slightly parted from the correct values. In this case the topology is much more irregular and presents several local minima as observed in Figure 2, which explains the higher difficulties of the ES to identify the correct values. These irregularities occur due to the use of two highly correlated measures, so the problem is "ill-posed". In the Figure it can be observed the existence of a range of parameters that practically conduct to the same error function value. Nonetheless, and even though the complex topology, the algorithm was able to identify values not far from the correct ones $(\mathrm{E}=2.196 \mathrm{GPa}$ and $\mathrm{K}_{0}=0.658$ ).

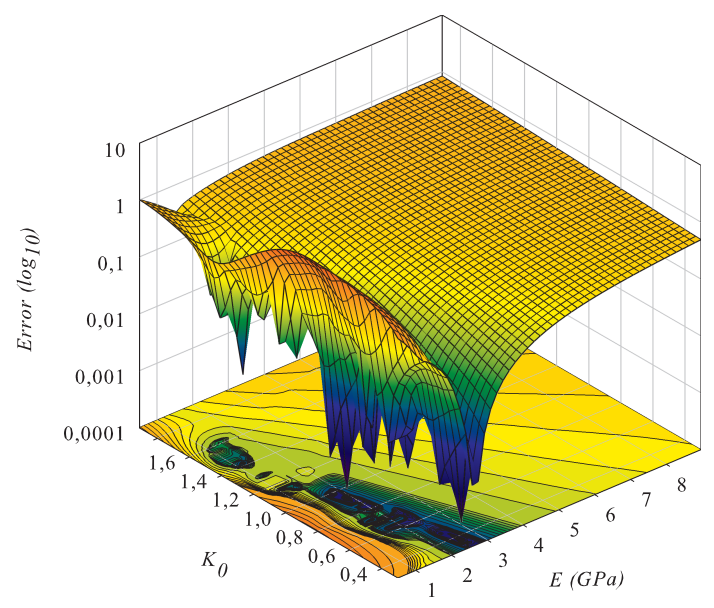

Figure 2. Topology of the error function for case 4

Using the elasto-plastic model, the complexity of the problem significantly increases due to the introduction of the non-linearity given by yielding zones forming in the surrounding formation which will affect the displacements. The goal was to identify the E, c' and $\phi$ ' (at once or in combinations of two) using different number of measurements. In Table 2 the identified values for each case are presented.

Table 2. Identified values for the elasto-plastic case

\begin{tabular}{llll}
\hline \multicolumn{3}{c}{ Parameter } \\
\hline Case & E $(\mathrm{GPa})$ & $\phi^{\prime}\left({ }^{\circ}\right)$ & $\mathrm{c}^{\prime}(\mathrm{kPa})$ \\
\hline 5 & 2.103 & 31.74 & 35.00 \\
6 & 2.028 & - & 48.18 \\
7 & 2.013 & 29.91 & - \\
8 & - & 30.33 & 47.46 \\
9 & 2.117 & 30.44 & 41.93 \\
10 & - & 31.06 & 43.56 \\
11 & 2.134 & 31.32 & 35.00 \\
\hline
\end{tabular}

In cases 5, 9 and 11 it was intended to identify the three parameters. The ES converged to the optimal solution in all cases demonstrating high robustness excepting for c' for which the identified values presented some deviation in relation to the correct one. In the worst case an error of approximately $22 \%$ was observed. This problem could be solved performing a higher number of calculations which would enhance the quality of the solution but also increase computational cost.

In Figure 3 the topology of the error function is presented for case 5 and for the parameters $\mathrm{E}$ and c'. The error function presents a very complex topology with several local minima but the algorithm was able to mitigate these difficulties. 

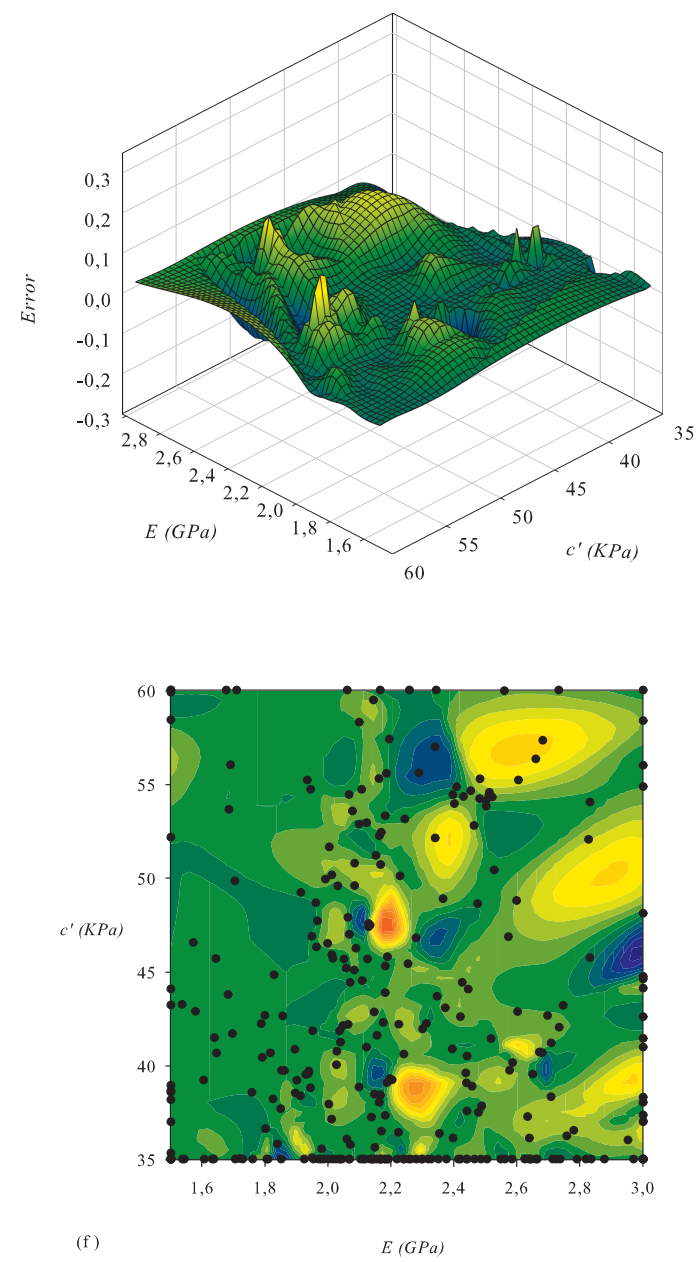

Figure 3. Topology of the error function for case 5 with parameters $\mathrm{E}$ and c'.

Cases 6, 7 and 8 consider combinations of the two geomechanical parameters with four displacement measurements while in case 10 it was intended to identify $\phi$ ' and c' using only two measurements. The algorithm converged to parameter values very near to the "real" ones. The only exception is the slight deviations for c' showing that this is the most difficult parameter to identify probably due to a lower influence or a more complex relation with the measured displacements that leads to higher irregularities in the error function topology.

Figure 4 shows the topologies of the error function for case 8 (cases 6 and 7 are similar). It can be observed a linear relation between the two strength parameters corresponding to an almost constant error function value. This "valley" in the error function crosses the point that represents the optimal solution and in this line several local minima exist which difficult convergence.
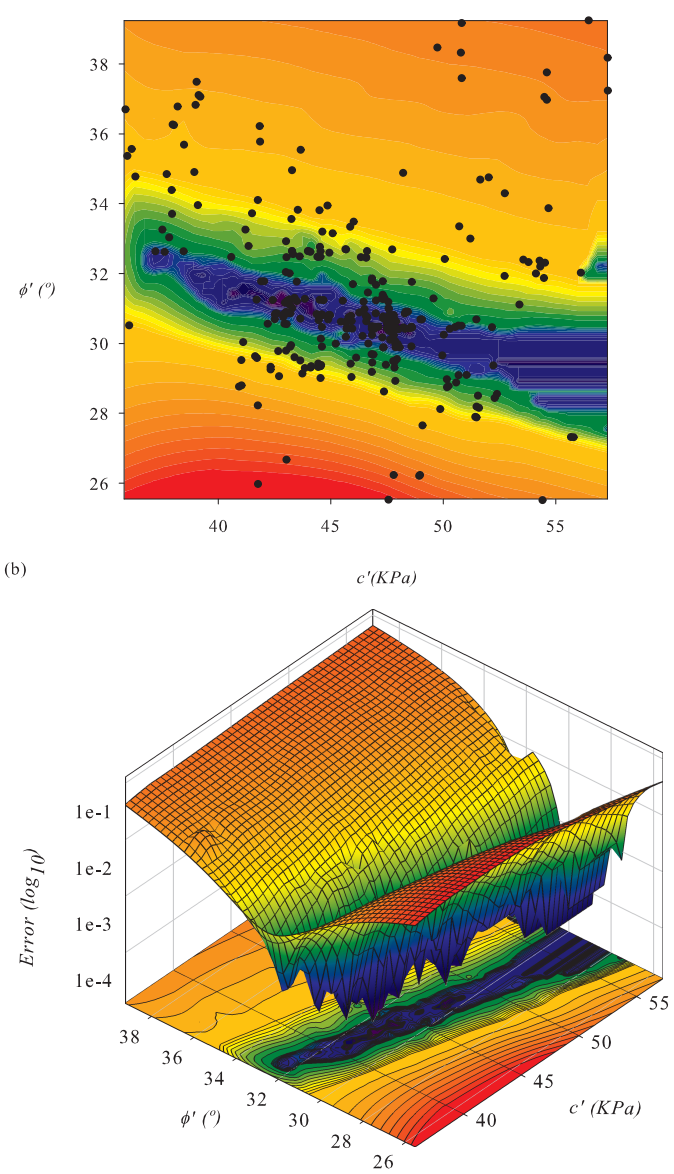

Figure 4. Topology of the error function for case 8 .

\section{CASE STUDY OF BEMPOSTA II}

\subsection{Hydraulic scheme and numerical model}

The Bemposta II is a new $191 \mathrm{MW}$ hydroelectric complex being built in the North of Portugal at the international stretch of Douro river in order to reinforce the power of an existing one (Bemposta).

The power plant of Bemposta II is composed by a vertical shaft excavated from the surface of the slope adjacent to the Bemposta dam (Lima et al., 2011). The excavation begins with a rectangular section, approximately $11 \mathrm{~m}$ depth, followed by an excavation with a circular cross-section with $22 \mathrm{~m}$ diameter and approximately $60 \mathrm{~m}$ deep. The circular cross section has a rectangular extension with horizontal dimensions of $16 \times 8.75 \mathrm{~m}^{2}$ (Figure 5).
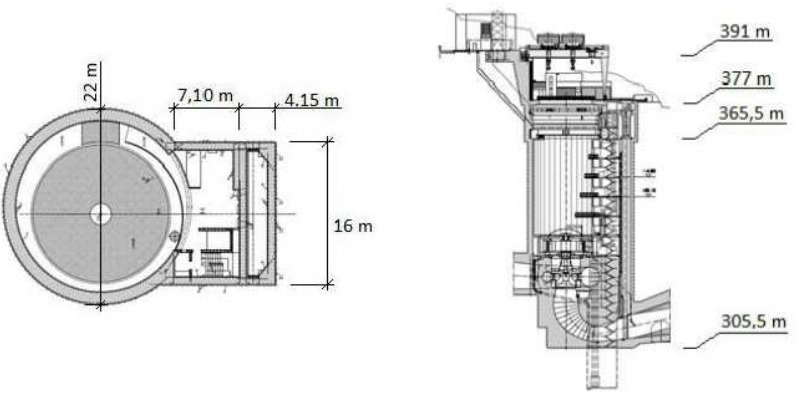

Figure 5 - Powerhouse shaft: section plan view (left) and hydraulic circuit and turbine axis vertical section (right). 
The Bemposta II scheme is located in highly metamorphosed heterogenous rock formations. The most part of the headrace tunnel was excavated in micaschist and migmatite while the powerhouse shaft and the tailrace tunnel, in good quality gneissic granite. The results of the in situ tests showed that the horizontal stress in the parallel direction to the slope is approximately 2.0 times higher than the vertical one. In the perpendicular direction the stress is approximately equal to the vertical one.

The monitoring data available at the time of the back analysis calculation was one horizontal doublerod extensometer (EV1). EV1 was anchored $11 \mathrm{~m}$ and $35 \mathrm{~m}$ (extensometers 1.11 and 1.35) into the rock mass from the excavation wall and it is located at level $367.5 \mathrm{~m}$ (Fig. 11). The maximum displacements measured were $0.33 \mathrm{~mm}$ and $1.02 \mathrm{~mm}$ respectively for extensometers 1.11 and 1.35 which are very low and point out to a good geomechanical quality of the rock mass.

The 3D numerical model of the power station of Bemposta II was developed using FLAC3D (Itasca, 2005) and simulates the construction process and the complex geometry of the shaft (Figure 6).

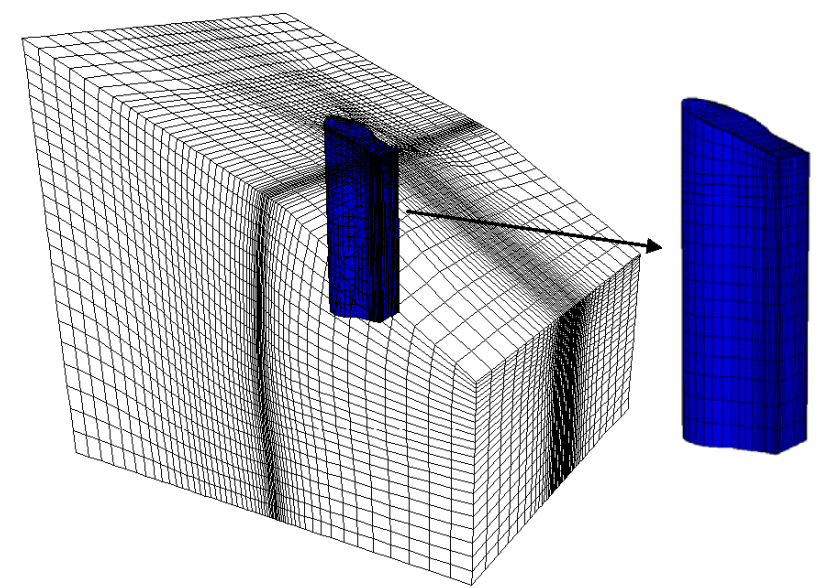

Figure 6 - 3D mesh developed for the Bemposta II powerhouse.

The Mohr-Coulomb failure criterion with nonassociated flow rule was used for the rock mass. According to the geologic and geotechnical survey, the following initial geomechanical parameters were set: $\phi^{\prime}=54^{\circ} ; c^{\prime}=2.65 \mathrm{MPa} ; \mathrm{E}=15 \mathrm{GPa}$ and $v=0.2$. The support system was composed by shotcrete and precasted concrete. The shotcrete was simulated using shell elements $0.20 \mathrm{~m}$ thick and the pre-casted concrete by volume elements. For both, a linear elastic and isotropic constitutive model with a Young modulus of $25 \mathrm{GPa}$ and $v$ of 0.2 was adopted.

In the numerical model the construction sequence was simplified and a total of six excavation stages were considered. In the preliminary calculations it was verified that this simplification did not affect considerably the results because the rock mass behavior is almost elastic.

\subsection{Results of back analysis}

For the back analysis process it was decided to identify $\mathrm{E}$ and $\mathrm{K}_{0}$ parallel to the slope direction which were the parameters with higher influence on the displacements.

To carry out the back analysis with the ES algorithm interval ranges for the parameters to identify must be set. The adopted ranges were the following: $\mathrm{E}[10 ; 60]$ and $\mathrm{K}_{0}[1 ; 4]$.

Figure 7 represents the topology of the error function (blue color in the Figure means lower values of the error). It shows that a linear relation between both parameters exists that leads practically to the same error values. Therefore the problem is "illposed" due to the fact that the two measurements are carried out in the same direction and they are highly correlated and compromise the uniqueness of the solution. These results corroborate the conclusions obtained previously in the synthetic case. To obtain a better definition of the error function it would be necessary to increase the monitoring data.

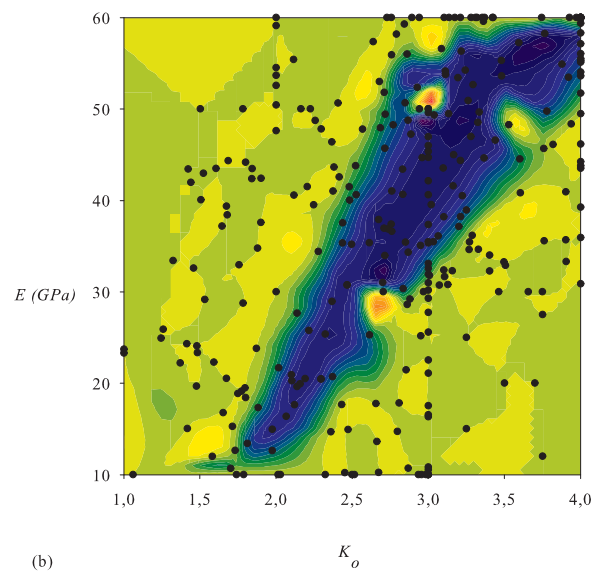

Figure 7 - Topology of error function for the case study.

In order to check the influence of the introduction of an additional extensometer in the back analysis process another synthetic study was carried out based on this real case. In this theoretical study an additional extensometer was considered, perpendicular to the existing one. The "real" displacements measured by the extensometers were calculated using the initial parameters $\mathrm{E}=30 \mathrm{GPa}$ and $\mathrm{K}_{0}=2.0$. These values are the optimal solution that the ES algorithm will search during the back analysis process. The ES algorithm was able to converge to the correct set of parameters showing high robustness in a total of 220 calculations.

Figure 8 shows the topology of the error function for this calculation. Comparing the topology of this error function with the previous one (Fig. 16) it can be concluded that the introduction of one additional extensometer improved the configuration of the error function and the definition of the optimal solution. A more accurate definition of the range of possible $\mathrm{K}_{0}$ values was achieved since in this case the valley of 
the error function is restricted to the interval [1.7; $2.2]$. For $E$ this interval is much wider, between approximately $25-35 \mathrm{GPa}$, in which the error value is practically constant. However, and in spite the complexity of the error function, the ES algorithm was able to identify the global minimum in an acceptable number of calculations.

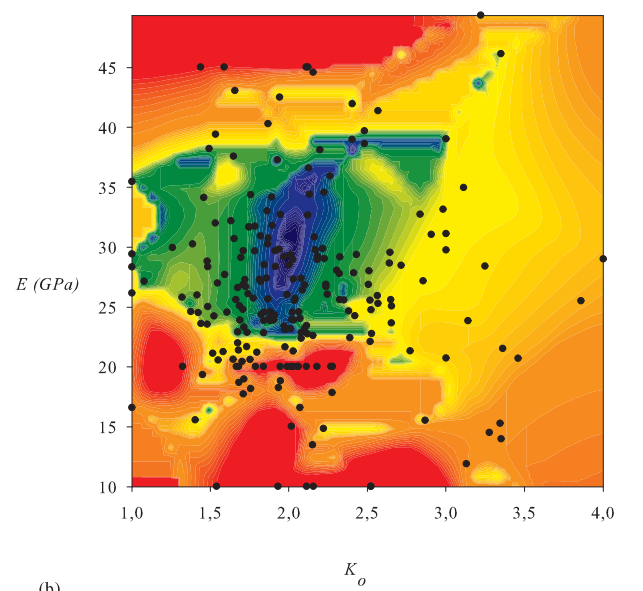

Figure 8 - Topology of the error function for the theoretical calculation.

\section{CONCLUSIONS}

As concluded in previous studies (e.g. Miranda, 2007) the classical optimization algorithms have a satisfactory performance in elasticity when the error function is strictly convex and presents a single minimum. In elasto-plasticity the problem becomes more complex due to the increased non-linearity provided by yielding zones near the excavation. In this situation the algorithms perform poorly since the error functions present very complex topologies.

The ES showed to be very robust even for highly complex error functions. The results for the elastic case showed that the ES was able to identify the correct values of the parameters in almost every case and in a manageable iteration number. The use of four measurements or only two with low correlation did not affect the results showing that with this algorithm it is possible to identify the elastic parameters with a very small amount of measurements. Only for the case of using two highly correlated measures the algorithm converged to slightly different values due to the high complexity of the error function.

Also in the elasto-plastic case the ES presented similar behavior. More difficulties arose in the identification of c' probably due to a lower influence or more complex relation with the measured values. However, good results were achieved, regardless of the high complexity of the error functions.

These results were achieved in a manageable number of calculations. This was a major issue in the past since evolutionary algorithms normally took thousands of calculations to converge which meant that with highly complex numerical models, they could lead to prohibitive computation times.

For the case study of Bemposta II powerhouse a back analysis process was carried out for the identification of $\mathrm{E}$ and $\mathrm{K}_{0}$. The back analysis process was not able to identify the set of optimal geomechanical parameters since the error function presented a constant value for a linear variation of the parameters. This was due to the limited available monitoring data. It was shown by a theoretical calculation that one additional extensometer would increase the definition of the error function topology and could allow the identification of the correct set of parameters.

\section{ACKNOWLEDGEMENTS}

This study was carried out under the framework of the strategic plan (2011-2013) of Territory, Environment and Construction Centre (C-TAC/UM), PEst-OE/ECI/UI4047/2011, approved by the FCT.

\section{REFERENCES}

Hoffmeister, F. and Bäck, T. 1991. Genetic Algorithms and evolution strategies: Similarities and differences. Lecture Notes in Computer Science, 1991, Volume 496/1991, 455469, DOI: 10.1007/BFb0029787.

Itasca. 2005. FLAC - Fast lagrangian analysis of continua. Version 5.0 User's manual, Minneapolis, USA.

Levasseur, S., Malécot, Y., Boulon, M. and Flavigny, E. 2010. Statistical inverse analysis based on genetic algorithm and principal component analysis: applications to excavation problems and pressuremeter tests. Int. Journal for Numerical and Analytical Methods in Geomechanics 2010; 34:471-491.

Lima, C., Resende, E., Esteves, C., Neves, J., 2011. Bemposta II Powerhouse Shaft. Geotechnical Characterization, Design and Construction. 12 ${ }^{\text {th }}$ Int. Congress on Rock Mechanics. October 19-22, Beijing, p. 6.

Matlab. 2009. The Language of Technical Computing Version 7.9. User Manual.

Miranda, T. 2007. Geomechanical parameters evaluation in underground structures. Artificial intelligence, Bayesian probabilities and inverse methods. PhD's thesis. University of Minho, Guimarães, Portugal, 291p.

Miranda, T., Eclaircy-Caudron, S., Dias, D., Gomes Correia, A, and Costa, L. 2011. Back analysis of geomechanical parameters by optimization of a 3D model of an underground structure. Tunnelling and Underground Space Tech., 26, 659-673.

Moreira, N., Miranda, T., Pinheiro, M., Fernandes, P., Dias, D., Costa, L., Sena-Cruz, J. 2013. Back analysis of geomechanical parameters in underground works using an Evolution Strategy algorithm. Tun. Underg. Space Tech., 33, 143-158.

Papon, A., Riou, Y., Dano, C. and Hicher, P-Y. 2012. Single and multi-objective genetic algorithm optimization for identifying soil parameters. Int. J. Numer. Anal. Meth. Geomech., 36(5), 597-618.

Rechenberg, I. 1973. Evolutionsstrategie: Optimierung technischer Systeme nach Prinzipien der biologischen Evolution. Friedrich Frommann Verlag, Stuttgart, 1973.

Rechenberg, I. 1994. Evolutionsstrategie'94. Stuttgard: Frommann-Holzboog.

Schwefel, H-P. 1995. Evolution and Optimal Seeking. John Wiley and Sons, 1995. 